\title{
BMJ Open Transport characteristics and predictors of mortality among neonates referred to a tertiary care centre in North India: a prospective observational study
}

\author{
Jasbir Singh (D) , ${ }^{1,2}$ Poonam Dalal, ${ }^{2}$ Geeta Gathwala, ${ }^{2}$ Ravi Rohilla ${ }^{3}$
}

To cite: Singh J, Dalal $P$, Gathwala G, et al. Transport characteristics and predictors of mortality among neonates referred to a tertiary care centre in North India: a prospective observational study. BMJ Open 2021;11:e044625. doi:10.1136/ bmjopen-2020-044625

- Prepublication history for this paper is available online. To view these files, please visit the journal online (http://dx.doi. org/10.1136/bmjopen-2020044625).

Received 09 September 2020 Accepted 02 June 2021

Check for updates

(C) Author(s) (or their employer(s)) 2021. Re-use permitted under CC BY-NC. No commercial re-use. See rights and permissions. Published by BMJ.

${ }^{1}$ Pediatrics, All India Institute of Medical Sciences, Bilaspur, Himachal Pradesh, India ${ }^{2}$ Pediatrics, Pt BD Sharma Post Graduate Institute of Medical Sciences, Rohtak, Haryana, India

${ }^{3}$ Community Medicine, Government Medical College and Hospital, Chandigarh, India

Correspondence to

Dr Jasbir Singh;

jasbir2001@gmail.com

\section{ABSTRACT}

Objective The paucity of specialised care in the peripheral areas of developing countries necessitates the referral of sick neonates to higher centres. Organised interhospital transport services provided by a skilled and wellequipped team can significantly improve the outcome. The present study evaluated the transport characteristics and predictors of mortality among neonates referred to a tertiary care centre in North India.

Design Prospective observational study.

Settings Tertiary care teaching hospital in North India. Patients 1013 neonates referred from peripheral health units.

Main outcome measures Mortality among referred neonates on admission to our centre.

Results Of the 1013 enrolled neonates, $83 \%$ were transferred through national ambulance services, $13.7 \%$ through private hospital ambulances and 3.3\% through personal vehicles. Major transfer indications were prematurity $(35 \%)$, requirement for ventilation $(32 \%)$, birth asphyxia (28\%) and hyperbilirubinaemia (19\%). Hypothermia (32.5\%, 330 of 1013), shock (19\%, 192 of 1013) and requirement for immediate cardiorespiratory support (ICRS) $(10.4 \%, 106$ of 1013) on arrival were the major complications observed during transfer. A total of $305(30.1 \%, N=1013)$ deaths occurred. Of these, $52 \%$ $(n=160)$ died within 24 hours of arrival. On multivariate logistic analysis, unsupervised pregnancy $(<4$ antenatal visits; $p=0.037)$, antenatal complications $(p<0.001)$, prematurity $\leq 30$ weeks $(p=0.005)$, shock $(p=0.001)$, hypothermia $(p<0.001)$, requirement for ICRS on arrival $(p<0.001)$, birth asphyxia $(p=0.004)$, travel time $>2$ hours $(p=0.005)$ and absence of trained staff during transfer $(p<0.001)$ were found to be significant predictors of mortality.

Conclusion The present study depicts high mortality among infants referred to our centre. Adequate training of peripheral health personnel and availability of prereferral stabilisation and dedicated interhospital transport teams for sick neonate transfers may prove valuable interventions for improved outcomes.

\section{INTRODUCTION}

Neonatal mortality rate (NMR) is a significant indicator of the socioeconomic and educational progress of a country and the efficacy
Strengths and limitations of this study

- Neonates were referred uniformly from the catchment area of our hospital, which is the largest government-funded tertiary centre in the state serving almost 10 million populations, and thus the sample may be considered representative of the whole study population.

- This study highlights the fact that endeavours to improve neonatal survival by setting up advanced neonatal intensive care units at referral centres will be a failure until dedicated efforts are done to improve the quality of primary care and sick patient transport.

- Neonates were enrolled for a period of 6 consecutive months and the comparison groups were made after the outcomes (ie, survival or death) have already occurred, so it is not likely to be subjected to any bias.

- We could not evaluate various neonatal sickness scores due to lack of diagnostic facilities and documentation from some of the referring centres.

- This was a single-centre study so the findings may not represent other parts of India.

of maternal-child healthcare policies. ${ }^{1}{ }^{2}$ In India, more than 25 million live births occur annually, with most of them occurring at district-level and lower-level hospitals. ${ }^{3}$ The scarcity of specialised care in the peripheral units necessitates the referral of sick neonates to higher centres. However, the frequency of adverse events during transfer is high due to suboptimal transport facilities. ${ }^{4}$ Ideally, all high-risk neonates should be transferred to referral centres in utero. However, the anticipation of perinatal complications before birth is not always possible ${ }^{56}$ Stabilisation of sick neonates before and during transfer can significantly increase the rate of survival. ${ }^{78}$

In India, free national ambulance services (NAS) were introduced for the transfer of sick mothers and children to government hospitals under the 'National Rural Health Mission/National Health Mission'. ${ }^{9}$ Haryana 
is a state in North India surrounding the national capital, New Delhi, and contributes to $1.37 \%$ of the total geographical area of the country. NAS has been providing referral transport coverage in Haryana since 2009. ${ }^{10}$ A significant proportion of women deliver at private health facilities. Apart from this, childbirths at home are still reported from many parts of the state. The interhospital transfer services of small-scale private hospitals are equipped differently and sometimes the use of personal vehicles may be required. Additionally, inadequate pre-referral stabilisation and interhospital communication are detrimental to ideal transfer services. ${ }^{11}$ Therefore, the present study attempted to examine the outcomes and characteristics of neonates transferred to our centre.

\section{METHODS}

\section{Study design and settings}

The present prospective observational studywas conducted at the Pandit Bhagwat Dayal Sharma Post Graduate Institute of Medical Sciences, Rohtak, Haryana, India, over 6 months from May 2016 to October 2016. Participation in the study was voluntary. The protocol was explained to the parents or guardians and informed consent was obtained before commencement of the study. Our institute is one of the largest government-funded medical colleges and referral centres in the region. Every year, approximately 1500-1700 outborn neonates are referred for admission from government and private hospitals distributed evenly in the catchment area. The referred neonates are first received in the emergency room (ER), where haemodynamic stabilisation and preliminary investigations are performed. The neonates are then admitted to the indoor patient department (IPD).

\section{Sample size}

Based on a preliminary analysis for 3 months prior to the study period, we have taken a liberal estimate of hospital mortality prevalence of $30 \%$, with a $10 \%$ relative error. The sample size was calculated using the following formula: $\mathrm{n}=\mathrm{zp}(1-\mathrm{p}) / \mathrm{d}^{2}$, where $\mathrm{z}$ is the standard normal deviate, $\mathrm{p}$ is the prevalence and $\mathrm{d}$ is the allowable error. The sample size calculated using the formula was found to be 933.33. We continued the present study for 6 consecutive months and enrolled a total of 1013 neonates. We have performed post-hoc power analysis using two-sided alpha level of 0.05 , which yielded sufficient power $(>80 \%)$, to find significant differences between survivors and deaths in the present study.

\section{Data collection}

Neonates (from birth to 28 days of life) referred for medical indications were enrolled in the study. Neonates whose parents or guardians refused to participate in the study or those referred for surgical indications were excluded. Government hospitals and sick newborn care units (SNCUs) use a predesigned referral card in which indications for transfer must be marked according to the International Classification of Diseases, 10th Revision and Clinical Modification, along with various therapeutic interventions administered before referral. Private healthcare institutes provide a referral summary containing details of the neonates and the treatment administered. Additionally, a detailed clinical history was obtained from the mother or accompanying guardians. The data were then recorded on a structured pro forma.

Data regarding the following factors were collected: (1) maternal factors: age, parity, status of antenatal care (ANC), risk factors, place, mode of delivery and associated complications; and (2) neonatal factors: baseline characteristics such as age, sex, gestational age at birth, birth weight and resuscitation requirement at birth (appearance, pulse, grimace, activity and respiration (APGAR) score whenever available); indication for referral; feeding details; haemodynamic status; presence of hypothermia, hyperbilirubinaemia, shock and hypoglycaemia on arrival at the ER (defined in table 1); and interventions performed immediately on arrival. Gestational age was assessed using the date of the last menstrual period and first-trimester ultrasonography, if available. In case of discrepancy, the new Ballard scoring was considered definitive for gestational age assessment. The first weight taken at a health facility was recorded as birth weight in cases of home delivery. (3) Transport characteristics were also collected and included documentation of vital signs before transfer, pre-referral interventions, modes of transport, time taken or distance travelled during transfer, availability of accompanying staff, and use of therapeutic or monitoring facilities. Each newborn was investigated and treated as per standard protocols and followed up until the final outcome, namely discharge from the hospital or death. At the end of the present study, we divided the neonates into two groups, (1) survivors and (2) deaths, and analysed their data to identify the predictors associated with mortality.

\section{Statistical analysis}

Continuous variables were analysed using percentage, mean and SD. Independent t-test was performed for comparison between normally distributed quantitative variables, whereas $\chi^{2}$ test was performed for qualitative variables. The authors initially performed a univariate analysis of the variables to study the association with the primary outcome. Thereafter, significant variables were used to create a model to predict the primary outcome measure (mortality). The multivariate analysis included stepwise logistic regression to select variables that caused the largest increase in the $\mathrm{R}^{2}$ value, and the association of the independent predictors with the dependent variable (survival of neonate) was analysed. The strength of the association was measured as OR with 95\% CI. The goodness of fit of the model was examined using Cox-Snell $R^{2}$ and Nagelkerke $R^{2}$. A p value of $<0.05$ was considered statistically significant. 
Table 1 Definition of various terminologies used in the study

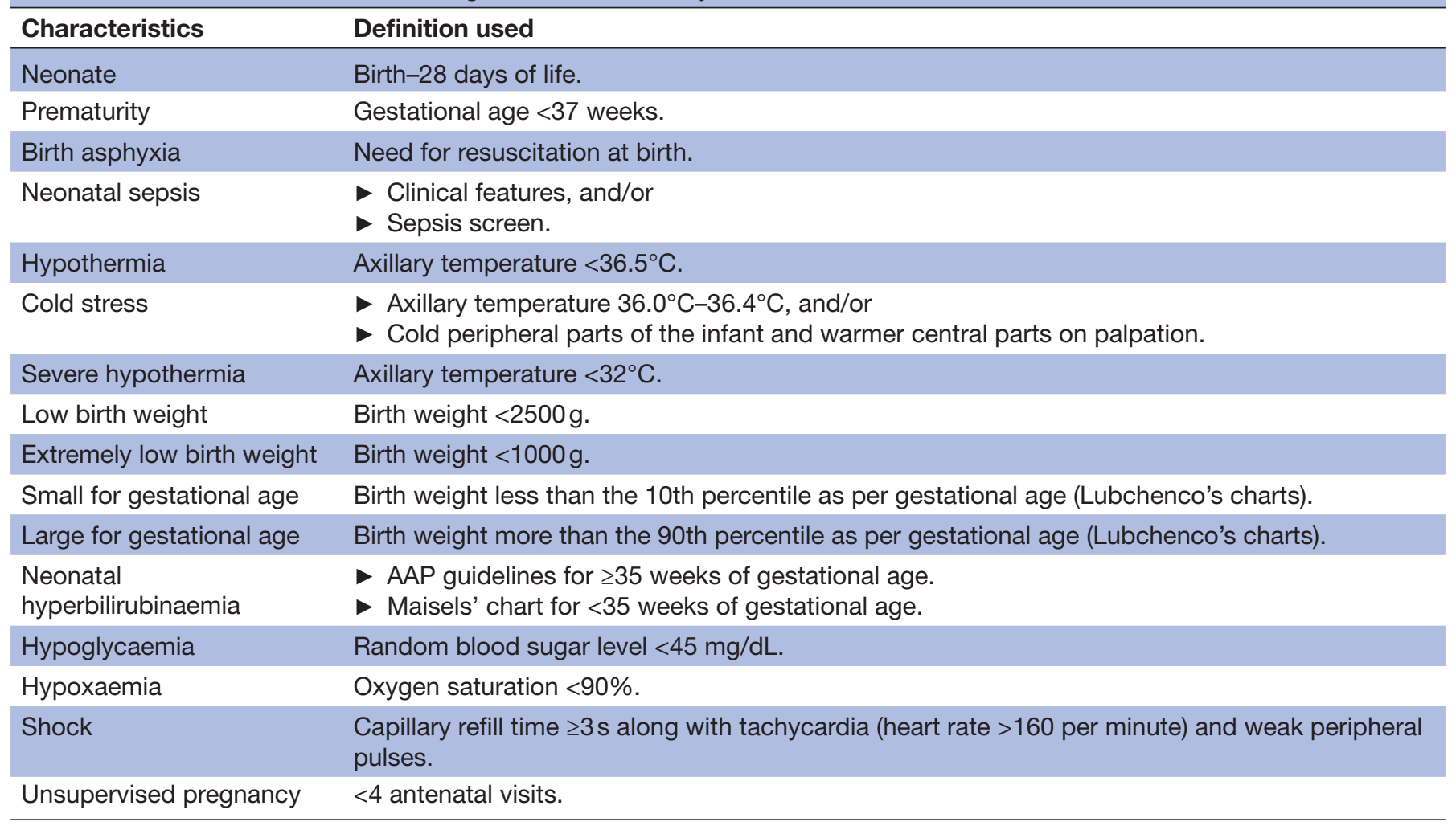

AAP, American Academy of Pediatrics.

Patients and public involvement statement

Patients and/or the public were not involved in the design, or conduct, or reporting or dissemination plans of this research.

\section{RESULTS}

\section{Clinical profile of referred neonates}

A total of 1240 referred neonates were admitted during the study. However, 227 neonates (including 148 neonates who were referred for surgical indications and 79 neonates whose parents or guardians refused to provide consent for participation in the study) were excluded from the study. Therefore, 1013 neonates were enrolled in the study, with a male to female ratio of $1.3: 1$. The mean gestational age and birth weight were $35.7 \pm 2.6$ weeks and $2212 \pm 699 \mathrm{~g}$, respectively. Pregnancy was supervised in $88.1 \%$ (893 of 1013 ) of women and $93.6 \%$ (949 of 1013) of neonates were born by vaginal deliveries. Table 2 presents the detailed clinical profile of the referred neonates.

\section{Transport characteristics}

Among 1013 neonates, the most common mode of transport was free-of-cost NAS $(83 \%)$, followed by private ambulances $(13.7 \%)$ and personal vehicles $(3.3 \%)$. Major transfer indications were prematurity (35\%), requirement for mechanical ventilation (32\%), birth asphyxia (28\%) and hyperbilirubinaemia (19\%). Of the total 1013 neonates, $61.8 \%$ got referred within the first 12 hours of life, $75.8 \%$ within 24 hours and $86 \%$ presented within the first week of life. Of the neonates $91 \%$ (926 of 1013) travelled for $\leq 2$ hours' duration or $\leq 100 \mathrm{~km}$. In the present study, 29.7\% (301 of 1013) of the mothers delivered at $\leq 34$ weeks gestation; however, none of them received antenatal steroids. Of the neonates $17 \%$ (172 of 1013) were administered vitamin $\mathrm{K}$ injection, and APGAR score was documented in only four neonates. At least one dose of intravenous antibiotics was administered in $47 \%$ of the neonates. Trained emergency staff accompanied $85 \%$ $(\mathrm{N}=1013)$ of the neonates during transfer.

\section{Haemodynamic status on admission to ER at our centre}

Among 1013 neonatal admissions, 32.5\% (330 of 1013) were hypothermic and 19\% (193 of 1013) were in a state of shock on arrival. Additionally, $63 \%$ of the neonates were already on intravenous fluid (IVF) and another $12 \%$ required IVF immediately on arrival. Endotracheal intubation was required in $10.4 \%$ (106 of 1013) of the neonates immediately on ER admission. Seizures were observed in $94(9.3 \%)$ neonates, whereas hypoglycaemia was observed in $7.4 \%$ (75 of 1013) of neonates on admission. Table 3 lists the details of the therapeutic interventions administered before transfer and within the first hour of arrival at the ER of our centre.

\section{Hospital course and predictors of mortality}

The present study observed 305 deaths among the 1013 enrolled neonates, resulting in an overall mortality rate of 
Open access

Table 2 Baseline characteristics of referred neonates and their mothers

\section{Characteristics}

$N=1013$

$\%$

Birth weight

$\geq 2500 \mathrm{~g}$

2000-2499g 218

$1500-1999 \mathrm{~g}$

$1000-1499 \mathrm{~g}$

$\leq 999 \mathrm{~g}$

32

448.3

21.5

17.2

Gestation

Term 614

Preterm

614

59.8

399

40.2

Sex

Male 581

Female

Antenatal period

Supervised pregnancies 893

88.1

Antenatal problem 389

38.4

Anaemia in mother

177

17.4

Leaking per vaginum (>24 hours)

75

7.4

Meconium-stained liquor

44

4.3

Pregnancy-induced hypertension

Multiple gestations

35

3.5

Prolonged labour

28

2.7

Rhesus incompatibility

22

2.2

Urinary tract infection

16

1.6

Hypothyroidism in mother

15

1.5

Antepartum haemorrhage

9

0.8

Obstructed labour

9

0.8

HIV+ mother

Pulmonary tuberculosis

0.6

6

4

3

0.4

0.3

Place of delivery

Government hospital

Private hospital

221

21.8

Home

39

3.8

Ambulance

9

0.9

Patients transferred according to age

At the initial 12 hours of life 627

At the initial 24 hours of life

768

61.8

768

75.8

At first week of life

Mode of delivery

Vaginal (vertex)

905

88.7

Lower segment caesarean section

64

2.6

Vaginal (breech)

Instrumental (forceps/vacuum)

38

1.6

Need for resuscitation at birth

6

6.9

288

32.5

Presenting features

Respiratory distress

669

66.1

Continued 


\begin{tabular}{lll}
\hline Table 2 Continued & & \\
\hline Characteristics & $\mathbf{N = 1 0 1 3}$ & \% \\
\hline Lethargy & 317 & 31.2 \\
\hline Delayed cry & 288 & 28.4 \\
Seizures & 136 & 13.4 \\
\hline Poor feeding/vomiting & 398 & 39.3 \\
\hline Jaundice & 189 & 19.8 \\
\hline Diarrhoea/weight loss & 104 & 10.2 \\
\hline Bleeding & 71 & 7.0 \\
Abdominal distension & 58 & 5.7 \\
Outcome & & 30.1 \\
\hline Death & 305 & 69.9 \\
\hline Discharge & 708 & \\
\hline
\end{tabular}

$30.1 \%$. Among these, $46 \%$ (140 of 305) of deaths occurred in the ER and the other $54 \%$ (165 of 305) occurred in the IPD. Of the total 305 deaths, $52 \%$ (160 of 305) died within $\leq 24$ hours of arrival at the ER. Of the neonates $70 \%$ (708 of 1013) survived and were discharged.

Univariate analysis was performed to compare survivors and deaths, and the significant mortality factors are depicted in table 4. The multivariate logistic analysis identified inadequate ANC, presence of antenatal

Table 3 Comparison of various interventions done at the referring facility (before transfer) and within the first hour of arrival at the ER of our centre

\begin{tabular}{|c|c|c|}
\hline Intervention & $\begin{array}{l}\text { Done at the } \\
\text { referring } \\
\text { facility } \\
\text { (before } \\
\text { transfer), n } \\
(\%)\end{array}$ & $\begin{array}{l}\text { Required } \\
\text { within the } \\
\text { first hour of } \\
\text { arrival at the } \\
\text { ER, } n(\%)\end{array}$ \\
\hline $\begin{array}{l}\text { Intravenous catheterisation } \\
(n=773)\end{array}$ & $647(83.7)$ & $126(16.3)$ \\
\hline $\begin{array}{l}\text { Antiepileptic drug } \\
\text { administration }(n=136)\end{array}$ & $42(30.9)$ & $94(69.1)$ \\
\hline Normal saline bolus $(n=215)$ & $22(10.2)$ & $193(89.8)$ \\
\hline $\begin{array}{l}\text { Dextrose bolus or infusion } \\
\text { rate increment }(n=86)\end{array}$ & $11(12.8)$ & $75(87.2)$ \\
\hline Dopamine infusion $(n=133)$ & $14(10.5)$ & $119(89.5)$ \\
\hline Dobutamine infusion $(n=62)$ & $4(6.5)$ & $58(93.5)$ \\
\hline Epinephrine infusion $(n=83)$ & $12(14.5)$ & $71(85.5)$ \\
\hline $\begin{array}{l}\text { Endotracheal intubation } \\
(n=150)\end{array}$ & $44(29.3)$ & $106(70.6)$ \\
\hline $\begin{array}{l}\text { Use of reservoir bag or } \\
\text { corrugated tube on AMBU } \\
(n=158)\end{array}$ & $8(5)$ & $150(95)$ \\
\hline Phototherapy (n=156) & $29(18.6)$ & $127(81.4)$ \\
\hline Total & 833 & 1089 \\
\hline
\end{tabular}

AMBU, artificial manual breathing unit; ER, emergency room. complications, preterm gestation ( $\leq 30$ weeks), delivery in the ambulance or transfer vehicles, presence of hypothermia and shock on arrival at the ER, requirement for endotracheal intubation, travel time $\geq 2$ hours, and absence of paramedical staff during transfer as significant factors associated with adverse outcome (table 5). The Cox-Snell $\mathbf{R}^{2}$ and Nagelkerke $\mathrm{R}^{2}$ indicated satisfactory goodness of fit of our model $(0.31 \%-0.66 \%)$ with an analysis of predictors of mortality (table 6 ).

\section{DISCUSSION}

The present study evaluated the transport characteristics and identified the predictors of mortality among referred neonates. Due to the large catchment area of our hospital, numerous neonates $(\mathrm{N}=1013 ; 5.6$ transfers per day) were referred during the study interval. The overall mortality rate was $30.1 \%$, and the majority of neonates experienced complications during transfer. The mortality among inborn neonates was $11 \%-12 \%$, and the transferred neonates needed more attention as evidenced by the higher rate of mortality. Studies from other parts of India and from resource-limited settings worldwide have described $10 \%-54 \%$ mortality among referred neonates. ${ }^{3}{ }^{6} 12-16$ The most commonly used mode of transfer was NAS, which has also been observed in studies from different parts of India and other lowerincome to middle-income countries (LMICs). 671316

The indications for neonatal referral differ across LMICs and developed countries. The major transfer indications for our cohort were prematurity, requirement for ventilation, birth asphyxia and hyperbilirubinaemia. These findings are concurrent with those of studies from other LMICs. ${ }^{5} 6$ 17-19 The National Neonatal-Perinatal Database network, a multicentre neonatal morbidity and mortality data collection initiative in India, described sepsis, birth asphyxia and prematurity as the major indications for referral. ${ }^{17}$ On the other hand, extreme prematurity and congenital malformations are reported as the major indications for referral in developed countries. ${ }^{20}$ 
Table 4 Comparison of baseline characteristics and clinical parameters between the two groups (univariate analysis)

\begin{tabular}{|c|c|c|c|}
\hline Parameters & Survivors (n=708), n (\%) & Deaths (n=305), n (\%) & $P$ value \\
\hline Maternal age (years) & $23.66 \pm 2.69$ & $24.15 \pm 2.84$ & 0.008 \\
\hline Unsupervised pregnancy & $63(8.89)$ & $57(18.68)$ & $<0.001$ \\
\hline Home delivery & $30(4.23)$ & $9(2.95)$ & 0.3216 \\
\hline Multiple gestations & $17(2.40)$ & $11(3.60)$ & 0.2852 \\
\hline Ambulance delivery & $4(0.56)$ & $5(1.63)$ & 0.001 \\
\hline Anaemia in mother & $89(12.57)$ & $88(28.85)$ & $<0.0001$ \\
\hline Leaking per vaginum & $42(5.9)$ & $33(10.8)$ & 0.006 \\
\hline Antenatal complications & $231(32.6)$ & $169(54.8)$ & $<0.0001$ \\
\hline Birth weight $(\mathrm{kg})$ & $2.34 \pm 0.62$ & $1.89 \pm 0.75$ & $<0.001$ \\
\hline Gestational age (weeks) & $36.37 \pm 2.00$ & $34.44 \pm 3.28$ & $<0.001$ \\
\hline Gestation $\leq 34$ weeks & $149(21.04)$ & $155(50.81)$ & $<0.0001$ \\
\hline Birth weight $<999 \mathrm{~g}$ & $6(0.47)$ & $26(8.88)$ & $<0.0001$ \\
\hline Need for resuscitation at birth & $194(27.40)$ & $95(31.14)$ & 0.226 \\
\hline Age at presentation (days) & $5.08 \pm 6.09$ & $2.28 \pm 3.63$ & 0.01 \\
\hline Sex & & & 0.495 \\
\hline Male & $411(58.05)$ & $170(55.73)$ & \\
\hline Female & 297 (41.94) & $135(44.26)$ & \\
\hline Hypothermia & & & $<0.001$ \\
\hline Cold stress & $123(17.34)$ & $22(6.28)$ & \\
\hline Hypothermia & $43(6.07)$ & $142(45.90)$ & \\
\hline Shock at presentation & $40(5.68)$ & $153(50.01)$ & $<0.001$ \\
\hline Hypoglycaemia & $42(5.93)$ & $33(10.08)$ & 0.006 \\
\hline Travel time $\geq 2$ hours & $43(6.0)$ & $44(14.4)$ & $<0.001$ \\
\hline Endotracheal intubation before referral & $16(2.25)$ & $50(16.39)$ & $<0.001$ \\
\hline $\begin{array}{l}\text { Endotracheal intubation immediately on arrival at the } \\
\text { emergency room }\end{array}$ & $7(0.98)$ & $99(32.45)$ & $<0.001$ \\
\hline Hospital stay (days) & $5.80 \pm 4.81$ & $0.48 \pm 0.33$ & $<0.001$ \\
\hline
\end{tabular}

Inadequate ANC and increased antenatal complications were associated with high neonatal mortality. In India, institutional births have increased from 39\% in 20052006 to $79 \%$ in $2015-2016 .{ }^{21}{ }^{22}$ However, the proportion of pregnant women receiving a minimum of four antenatal visits has increased modestly from $37.0 \%$ to $51.2 \%$, which is considerably lower than the global average of $61.8 \% .{ }^{22} 23$ Due to the wide variations in geographical and socioeconomic profiles of populations in India, achieving full ANC coverage remains challenging. Therefore, the Indian government has initiated a cash incentive scheme for pregnant women who register themselves at health centres. ${ }^{24}$ Private hospitals are preferred for delivery by several patients due to better privacy and less crowding. Thus, active participation of these facilities in the maternal and child health programmes is required.

Gestational age at birth and birth weight are the most vital determinants of neonatal survival, especially in LMICs. ${ }^{25}$ Low socioeconomic profile, poor nutritional status of mothers, short interpregnancy interval and increased antenatal complications may be factors that contribute to increased proportion of preterm and low birthweight (LBW) neonates. Another reason for the high preterm and LBW infants load may be due to our hospital being a referral centre. In our series, out of 305 deaths, 69\% (213 of 305) were LBW and 50\% (155 of 305 ) of infants were of $\leq 34$ weeks gestation. Inadequate use of antenatal steroids may be another factor contributing to the high mortality among premature infants in our cohort.

The significance of pre-referral stabilisation is well documented in the literature. ${ }^{26}$ Despite travel durations being $\leq 2$ hours in most of the neonates in our cohort, $52 \%$ of deaths were reported within 24 hours of arrival as the neonates were too sick to refer. Further, the deterioration of a sick patient during transfer may be completely unrelated to the severity of the primary illness. ${ }^{14}$ Thus, adequate training of the accompanying medical staff in basic resuscitation skills cannot be overemphasised. The predictors with the highest influence on mortality in the present study can be mitigated by simple interventions such as provision of IVF, temperature maintenance, 
Table 5 Independent predictors of neonatal mortality (by stepwise multivariate logistic regression)

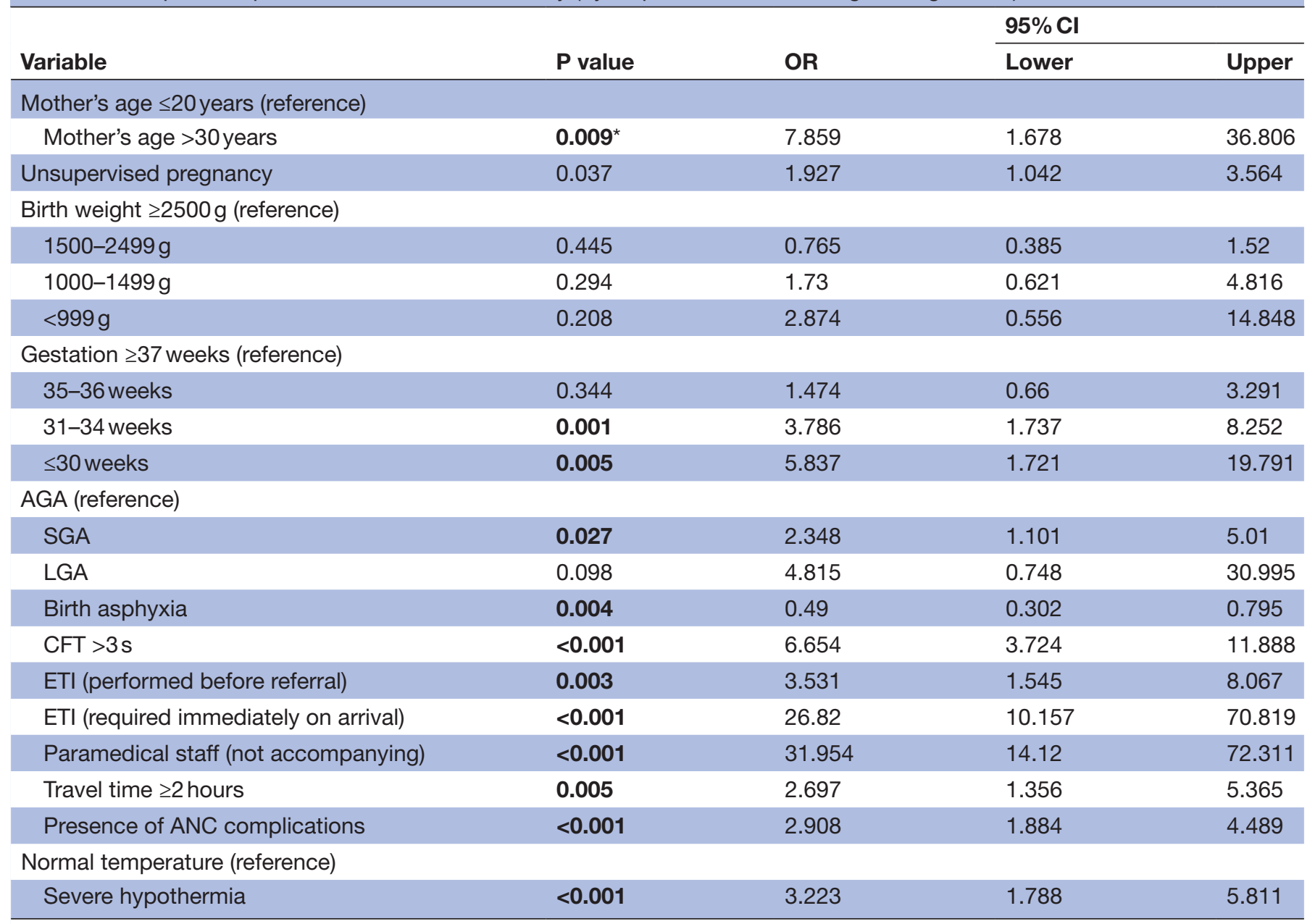

Reference category has been mentioned for each variable.

*significant $p$-value

AGA, appropriate for gestational age; ANC, antenatal care; CFT, capillary filling time; ETI, endotracheal intubation; LGA, large for gestational age; SGA, small for gestational age.

neonatal resuscitation skills and ensuring that trained paramedical staff accompanied the patient. These findings have been mirrored in two other studies at Indian centres. ${ }^{27}{ }^{28}$ Using a well-organised sick patient transfer system, Kumutha et al described the Gunapati Venkata Krishna Reddy Emergency Management and Research Institute model providing quality and efficient services in the southern part of India. ${ }^{8}$ In our region, the NAS staff are trained to provide basic life support, international trauma life support and advanced life support in obstetrics, but training for specific issues related to sick neonates and young infants transport is to be achieved. ${ }^{10}$ We recommend that training of sick neonate transfer teams in our region should focus more on imparting skills in adequate neonatal assessment, pre-referral stabilisation and management of adverse events during transfer. The primary care provider at the referring hospital should communicate with the specialist at the referral centre and inform the need for transfer and the condition of the neonate before commencing transport.
Several simple programmes such as TOPS (temperature, oxygenation, perfusion and sugar) and STABLE (sugar, temperature, airway, blood pressure, laboratory investigations and emotional support to family) can be used to guide adequacy of pre-referral stabilisation. ${ }^{29} 30$ In addition to making provisions for transport incubators, use of an extra layer of clothing, with head cap and socks, and providing kangaroo mother care (KMC) can be implemented to maintain the temperature of the neonate during transfer. Airway and breathing management and adequate oxygenation and perfusion among sick neonates are crucial. Endotracheal intubation must be performed before commencing transport if severe respiratory compromise is anticipated. Modular transport incubators with inbuilt ventilator devices can be incorporated into ambulances. Artificial manual breathing units with differently sized masks must be readily available on all transports, regardless of an infant's respiratory status. Skills regarding the establishment of peripheral intravenous access must be refined among peripheral health 


\begin{tabular}{|c|c|c|c|c|}
\hline Step & Variable & -2 log likelihood & Cox-Snell $\mathbf{R}^{2}$ & Nagelkerke $\mathbf{R}^{2}$ \\
\hline 1 & Capillary filling time & 982.368 & 0.222 & 0.315 \\
\hline 2 & Accompanying staff & 875.995 & 0.300 & 0.425 \\
\hline 3 & ETI on arrival at the ER & 780.411 & 0.363 & 0.514 \\
\hline 4 & Birth weight & 710.059 & 0.406 & 0.575 \\
\hline 5 & Temperature & 679.100 & 0.424 & 0.601 \\
\hline 6 & ANC complications & 651.859 & 0.439 & 0.622 \\
\hline 7 & Birth asphyxia & 644.745 & 0.443 & 0.628 \\
\hline 8 & ETI before referral & 637.994 & 0.447 & 0.633 \\
\hline 9 & Travel time & 630.999 & 0.451 & 0.638 \\
\hline 10 & Mother's age & 621.980 & 0.455 & 0.645 \\
\hline 11 & Gestation period & 613.573 & 0.460 & 0.652 \\
\hline 12 & AGA & 606.671 & 0.464 & 0.657 \\
\hline 13 & Numbers of ANC visits & 602.441 & 0.466 & 0.660 \\
\hline
\end{tabular}

AGA, appropriate for gestational age; ANC, antenatal care; ER, emergency room; ETI, endotracheal intubation.

workers. Provision of syringe infusion pumps will ensure the administration of a calculated volume of IVF during transfer. Temperature, oxygen saturation, ECG and perfusion index can be monitored with multichannel monitors during transfer. Real-time interaction between tertiary centres and transfer teams can be made feasible using integrated wireless cellular technology and through telemedicine departments for better coordination.

As acknowledged globally, the establishment of SNCUs at the periphery to provide level II neonatal intensive care is a crucial step to reducing the high NMR in India. ${ }^{31}$ In the present study, $25 \%$ of the neonates with hyperbilirubinaemia and $8 \%$ of the LBW neonates were discharged from our centre after 48-72 hours. These patients can be managed at SNCUs with measures such as early establishment of breast feeding, phototherapy and KMC. However, owing to the remoteness of SNCUs, maintenance of adequate infrastructure remains challenging. ${ }^{32}$ India has a long way to go in terms of ensuring easily accessible ideal healthcare to every neonate. The most commonly encountered challenges are as follows: (1) ill-equipped health facilities, (2) lack of awareness and skills among peripheral health workers, and (3) poor implementation of various referral guidelines such as the Integrated Management of Neonatal and Childhood Illness. In addition to establishing neonatal intensive care units, the present scenario can be improved by increasing community awareness and participation, such as involvement of local rural healthcare workers, promotion of home-based newborn care and exploring non-governmental organisation support.

The main strength of the present study was the enrolment of neonates uniformly referred from the catchment area of our hospital, which is the single largest government-funded tertiary care centre in the region. Thus, the sample may be considered representative of the population. However, the present study also has certain limitations. The data were collected for 6 months at a single centre in North India and the results may not represent other parts of the country. As peripheral referral centres lacked diagnostic facilities, limited data availability precluded us from evaluating various neonatal sickness scores. Further, the data may be several years old. However, this is the first study done after the launch of NAS in our region which provided insight into neonatal transport services to a hospital with a high case load.

\section{CONCLUSION}

The present study depicts the haemodynamic worsening of neonates and the high mortality rate during referral and transport. Adequate pre-referral stabilisation, increased awareness, enhanced basic resuscitation skills of the peripheral health staff and availability of appropriate equipment at SNCUs can improve the outcomes of the referred neonates in our region.

Acknowledgements The authors are very thankful to the parents of the neonates who participated in the study.

Contributors JS developed the idea of the study and prepared the first draft with PD. JS, PD and GG contributed substantially to the research protocol. JS,PD and RR led the data analysis or interpretation of data for the work. JS wrote the draft of the manuscript and revised it critically for important intellectual content, with significant contributions from PD, GG and RR. All authors approved the final version of the manuscript.

Funding The authors have not declared a specific grant for this research from any funding agency in the public, commercial or not-for-profit sectors.

Competing interests None declared.

Patient and public involvement Patients and/or the public were not involved in the design, or conduct, or reporting, or dissemination plans of this research.

Patient consent for publication Not required.

Ethics approval This study was done after obtaining approval from the Departmental Ethics Committee, Pediatrics Department, Pandit Bhagwat Dayal 
Sharma Post Graduate Institute of Medical Sciences, Rohtak, Haryana, India. The study was conducted in accordance with the principles of the Declaration of Helsinki and the International Council on Harmonization Guidelines for Good Clinical Practice. Anonymity of the enrolled patients in the manuscript was maintained.

Provenance and peer review Not commissioned; externally peer reviewed.

Data availability statement All data relevant to the study are included in the article or uploaded as supplementary information.

Open access This is an open access article distributed in accordance with the Creative Commons Attribution Non Commercial (CC BY-NC 4.0) license, which permits others to distribute, remix, adapt, build upon this work non-commercially, and license their derivative works on different terms, provided the original work is properly cited, appropriate credit is given, any changes made indicated, and the use is non-commercial. See: http://creativecommons.org/licenses/by-nc/4.0/.

ORCID iD

Jasbir Singh http://orcid.org/0000-0001-7721-5614

\section{REFERENCES}

1 Neonatal Mortality Rate. The global health OBSERVATORY. indicator metadata registry list, 2021. Available: https://www.who.int/data/gho/ indicator-metadata-registry/imr-details/67

2 Yu VY, Global YVY. Global, regional and national perinatal and neonatal mortality. J Perinat Med 2003;31:376-9.

3 Zodpey S, Paul VK, eds. PHFI, AlIMS, SC: state of India's neonates (SOIN). New Delhi, India: Public Health Foundation of India, All India Institute of Medical Sciences and Save the Children, 2014. https:// www.newbornwhocc.org/SOIN_PRINTED\%2014-9-2014.pdf

4 Kumar PP, Kumar CD, Shaik FAR, et al. Prolonged neonatal interhospital transport on road: relevance for developing countries. Indian J Pediatr 2010;77:151-4.

5 Sabzehei MK, Basiri B, Shoukohi M. Factors affecting the complications of interhospital transfer of neonates referred to the neonatal intensive care unit of Besat hospital in 2012-2013. J Clin Neonatol 2016;5:238-42.

6 Jajoo M, Kumar D, Dabas V, et al. Neonatal transport: the long drive has not even begun. Indian J Community Med 2017;42:244-5.

7 Kumar PP, Kumar CD, Shaik F, et al. Transported neonates by a specialist team - how STABLE are they. Indian J Pediatr 2011;78:860-2.

8 Kumutha J, Rao GVR, Sridhar BN, et al. The GVK EMRI maternal and neonatal transport system in India: a mega plan for a mammoth problem. Semin Fetal Neonatal Med 2015;20:326-34.

9 Janani Shishu Suraksha Karyakaram (JSSK). National health mission (Ministry of health and family welfare, government of India). $\mathrm{RMNCH}+\mathrm{A}, 2020$. Available: https://nhm.gov.in

10 National Health Mission, Haryana. Referral transport, 2020. Available: http://www.nhmharyana.gov.in/programDetails?id=14

11 Jayashree M, Nallasamy K, Khandelwal N, et al. Feasibility of an e-Referral system for streamlining referrals to pediatric emergency room of a tertiary care teaching hospital in North India- a preliminary report. J Pediatr Crit Care 2018;5:99-102.
12 Aggarwal KC, Gupta R, Sharma S, et al. Mortality in newborns referred to tertiary Hospital: an introspection. J Family Med Prim Care 2015;4:435-8.

13 Baidya M, Shirin M, Saha LC. Transport factors affecting the outcome of referred neonates admitted in a tertiary care hospital. Bangladesh J Child Health 2017;41:159-64.

14 Rathod D, Adhisivam B, Bhat BV. Transport of sick neonates to a tertiary care Hospital, South India: condition at arrival and outcome. Trop Doct 2015;45:96-9.

15 Henry S, Trotman H. Challenges in neonatal transport in Jamaica: a resource-limited setting. J Trop Pediatr 2017;63:307-13.

16 Abdulraheem MA, Tongo OO, Orimadegun AE, et al. Neonatal transport practices in Ibadan, Nigeria. Pan Afr Med J 2016;24:216.

17 National Neonatology Forum (NNPD Network), India. Neonatal morbidity and mortality: report of the National neonatal perinatal database, 2005. Available: https://www.newbornwhocc.org/pdf/ nnpd_report_2002-03.PDF

18 Dey SK, Sharker S, Jahan I, et al. Neonatal transport - experience of a tertiary care hospital of Bangladesh. Mymensingh Med $J$ 2017;26:169-74.

19 Gurubacharya SM, Gurubacharya RL. An overview of neonatal admissions at college of medical sciences (COMS). J. Nepal Paedtr. Soc. 2009;27:73-4.

20 Simpson CDA, Ye XY, Hellmann J, et al. Trends in causespecific mortality at a Canadian outborn NICU. Pediatrics 2010;126:e1538-44.

21 International Institute for Population Sciences (IIPS) and ICF. National family health survey (NFHS-4), 2015-16. Mumbai: IIPS, 2017.

22 Vellakkal S, Gupta A, Khan Z, et al. Has India's national rural health mission reduced inequities in maternal health services? A pre-post repeated cross-sectional study. Health Policy Plan 2017;32:79-90.

23 Kumar G, Choudhary TS, Srivastava A, et al. Utilisation, equity and determinants of full antenatal care in India: analysis from the National family health survey 4. BMC Pregnancy Childbirth 2019;19:327.

24 Ministry of Women \& child development, Government of India. Pradhan Mantri Matru Vandana Yojana, 2020. Available: https://wcd. nic.in/schemes/pradhan-mantri-matru-vandana-yojana

25 Sankar MJ, Neogi SB, Sharma J, et al. State of newborn health in India. J Perinatol 2016;36:S3-8.

26 Gente M, Lallo DD, Franco F, et al. Stabilization of the critically ill neonate awaiting transport. Ital J Pediatr 2015;41:A15.

27 Narang M, Kaushik JS, Sharma AK, et al. Predictors of mortality among the neonates transported to referral centre in Delhi, India. Indian J Public Health 2013;57:100-4.

28 Sehgal A, Roy MS, Dubey NK, et al. Factors contributing to outcome in newborns delivered out of hospital and referred to a teaching institution. Indian Pediatr 2001;38:1289-94.

29 Mathur NB, Arora D. Role of tops (a simplified assessment of neonatal acute physiology) in predicting mortality in transported neonates. Acta Paediatr 2007;96:172-5.

30 Taylor RM, Price-Douglas W. The S.T.A.B.L.E. program: postresuscitation/pretransport stabilization care of sick infants. $J$ Perinat Neonatal Nurs 2008;22:159-65.

31 Neogi SB, Malhotra S, Zodpey S, et al. Assessment of special care newborn units in India. $J$ Health Popul Nutr 2011;29:500-9.

32 Prinja S, Manchanda N, Mohan P, et al. Cost of neonatal intensive care delivered through district level public hospitals in India. Indian Pediatr 2013;50:839-46. 